\title{
Design of low-pass fractional order IIR digital filters
}

\author{
Dharmendra K. Upadhyay* and Prachi Srivastava
}

\author{
Division of ECE, Netaji Subhas Institute of Technology, Dwarka, New Delhi-110078, India \\ *Email: upadhyay1975.d@gmail.com
}

Traditionally, the designing of an IIR digital filter, the method used is to design an analog filter and then converting it to the digital filter by performing optimum s-to-z transformation. In most of the cases, the analog filter order comes in fraction to meet the required specifications, which is approximated to the next higher integer. Therefore, the performance of IIR filter is also limited to the integer order only.

Recently, lot of work has been done in the field of analog filter realization in fractional domain [1] and the proposal of fractional order differentiator Models [2-4]. Therefore, this paper presents the steps to design a fractional order IIR digital filter by applying different s-to$\mathrm{z}$ transformations. In the first step of designing procedure, a fractional order analog filter is designed, which act as the analog prototype for designing the fractional order IIR digital filter.

The next step is to select the first order s-to-z operator based on the mapping between s-to-z domains. For this three different first order s-to$\mathrm{z}$ operators are discussed and compared in this paper. Figure 1 shows the comparison of magnitude response for all three s-to-z operators with the ideal one. It is clear that the optimized Al-Alaoui operator approximates best to the ideal one.

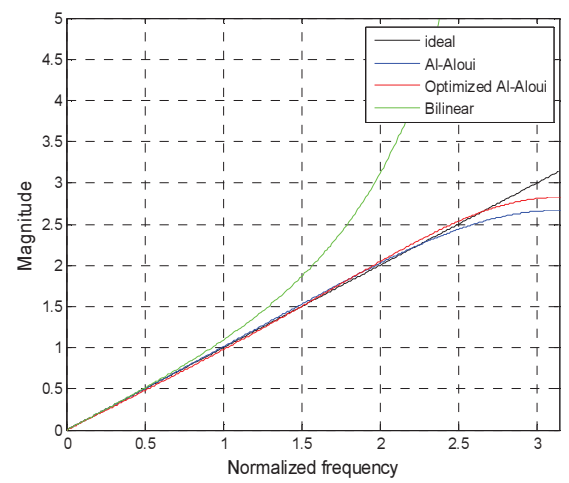

Figure 1: Comparison of magnitude responses of different s-to-z operators with the ideal one

These operators are used to convert analog prototype into the digital filters. Simulation results show that the bilinear transformation gives the sharpest transient band and gives the filter response, which is closer to the ideal one. However, the Al-Alaoui and optimized AlAlaoui s-to-z operators give quite similar performance with respect to the analog prototype filter, in which optimized Al-Alaoui operator gives comparatively better performance in these two. Figure 2 shows the magnitude responses for designed fractional order low-pass digital filters for different pass edge digital frequencies using bilinear transformation.

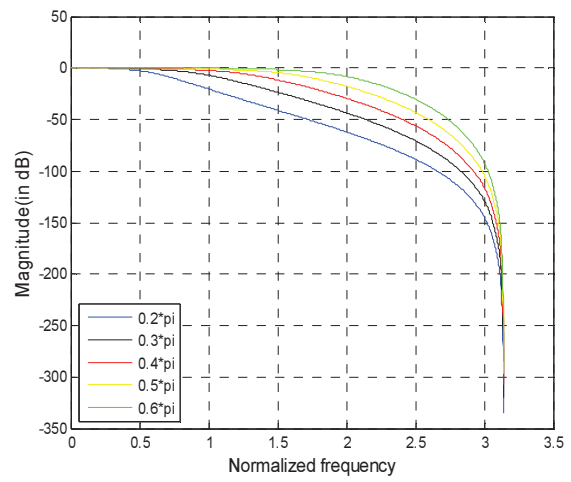

Figure 2: Magnitude responses of designed fractional order low-pass digital filters for different pass edge frequencies using bilinear transformation

\section{References}

1. A.S. Ali, A.G. Radwan, and A.M. Soliman, "Fractional Order Butterworth Filter: Active and Passive Realizations," in Emerging and Selected Topics in Circuits and Systems, IEEE Journal, vol. 3, no. 3, pp. 346-354, Sept. 2013.

2. M. Gupta, and R. Yadav, "New Improved Fractional Order Differentiator Models Based on Optimized Digital Differentiators," The Scientific World Journal, vol. 2014, pp.1-11, Feb. 2014.

3. M.A. Al-Alaoui, "Al-Alaoui operator and the new transformation polynomials for discretization of analogue systems." Electrical Engineering, Springer, vol. 90, no. 6, pp. 455-467, Jan. 2008.

4. Y.Q. Chen, and B.M. Vinagre, "A new IIR-type digital fractional order differentiator," Signal Processing, vol. 83, no. 11, pp.2359-2365, Nov. 2003. 